1998-01-01

\title{
Endomorphism Rings and Automorphism Groups of Separable Torsion-Free Modules Over Valuation Domains
}

\author{
Brendan Goldsmith \\ Technological University Dublin, brendan.goldsmith@tudublin.ie \\ P. Zanardo \\ University of Padova
}

Follow this and additional works at: https://arrow.tudublin.ie/scschmatbk

Part of the Mathematics Commons

\section{Recommended Citation}

Goldsmith, B. \& Zanardo, P. (1998). Endomorphism Rings and Automorphism Groups of Separable Torsion-Free Modules over Valuation Domains. Abelian groups, Module Theory, and Topology, CRC Press. doi:10.21427/kpjy-9j54

This Book Chapter is brought to you for free and open access by the School of Mathematics at ARROW@TU Dublin. It has been accepted for inclusion in Book chapter/book by an authorized administrator of ARROW@TU Dublin. For more information, please contact arrow.admin@tudublin.ie, aisling.coyne@tudublin.ie, gerard.connolly@tudublin.ie. 


\title{
ENDOMORPHISM RINGS AND AUTOMORPHISM GROUPS OF SEPARABLE TORSION-FREE MODULES OVER VALUATION DOMAINS
}

\author{
B. Goldsmith and P. Zanardo
}

\section{INTRODUCTION}

In recent work Corner and Goldsmith $[\mathrm{CG}]$ have shown that the automorphism group of a reduced torsion-free $p$-adic module $(p \neq 2)$ determines the module, i.e. if $G, G^{\prime}$ are such modules with isomorphic automorphism groups, $\operatorname{Aut}(G) \cong \operatorname{Aut}\left(G^{\prime}\right)$, then $G$ and $G^{\prime}$ are isomorphic as $p$-adic modules. Their result is but one of a number of results relating the structure of the endomorphism ring or automorphism group of a module to the structure of the module itself: see $[\mathrm{K}],[\mathrm{H}],[\mathrm{Li}],[\mathrm{Le}],[\mathrm{R}]$ and $[\mathrm{W}]$ for related results on various classes of modules.

The present work seeks to generalise the results in $[\mathrm{CG}]$ and $[\mathrm{W}]$ : the ring of $p$-adic integers is replaced by a more general valuation domain. However, one realizes that extensions of results may be achieved only if one confines oneself to a particular, important class of valuation domains, the so-called totally branched discrete valuation domains (see [Z] and $\S 1$ ). The price to be paid for such a significant generalisation is that it is necessary to restrict attention to torsion-free separable modules. Recall that a torsion-free module $M$ over a valuation domain $R$ is said to be separable if every element of $M$ is contained in a direct summand of $M$ of rank one (which is necessarily isomorphic either to an ideal or to the field of fractions of $R$ ). It is well known that torsion-free $p$-adic modules are automatically separable (see $[\mathrm{K}])$.

In $\S 1$ we focus attention on endomorphism rings of separable modules over valuation domains. Our principal result in this section is that totally branched discrete valuation domains are characterised by the property that their separable torsionfree modules are endomorphically unique, i.e. if $M, M^{\prime}$ are separable torsion-free modules with $\operatorname{End}_{R} M=\operatorname{End}_{R} M^{\prime}$, then $M \cong M^{\prime}$.

In $\S 2$ we focus on automorphism groups and, inevitably, we have as a blanket hypothesis that 2 is a unit of the base $\operatorname{ring} R$. The results obtained are, not surprisingly, weaker that the corresponding results on vector spaces or $p$ adic modules. Specifically we need to replace isomorphism between $R$-modules by semi-isomorphism. Recall that $R$-modules $M, M^{\prime}$ are semi-isomorphic if there is a ring automorphism $g$ of $R$ and an additive bijection $\phi: M \rightarrow M^{\prime}$ such that $\phi(r x)=g(r) \phi(x)$ for all $r \in R$ and $x \in M$.

Our main theorem in the second section extends Theorem 1 of [CG]. Some restrictions on the hypotheses naturally arise. We have to consider separable torsion-free modules over a totally branched discrete valuation domain $R$ satisfying the further property that the the rank-one $R$-modules are determined by their automorphism 
groups (otherwise our approach is doomed: see Proposition 2.5). Let $G, \widetilde{G}$ be two such $R$-modules and suppose that there is an isomorphism $\Phi: \operatorname{Aut}(G) \rightarrow \operatorname{Aut}(\widetilde{G})$. Let $R_{J}$ be the largest localization of $R$ such that $G, \widetilde{G}$ are $R_{J}$-modules. Then $\Phi$ determines an automorphism $g$ of $R_{J}$ such that $G$ is $g$-semi-isomorphic to either $\widetilde{G}$ or to $\operatorname{Hom}\left(\widetilde{G}, R_{J}\right)$, as $R_{J}$-modules.

The notion of semi-isomorphism occurs in the analogous classical problem for vector spaces (see $[\mathrm{B}]$ ), but for vector spaces (or more generally free modules) it is easy to see that semi-isomorphism always implies isomorphism. We are unable to determine whether such an implication is valid in our general context. We can however recover isomorphism for modules over the discrete valuation rings of rank one which lie between $\mathbb{Z}_{p}$ and $\hat{\mathbb{Z}}_{p}$ (Theorem 2.3).

\section{§1. ENDOMORPHISM RINGS}

In what follows, unless stated to the contrary, $R$ will denote a valuation domain, $P$ its maximal ideal, and $U(R)$ the multiplicative group of the units of $R$. For general definitions and results on valuation domains and their modules we refer to the book by Fuchs and Salce [FS]. Our notation is standard, in accord with $[\mathrm{F}]$ and [FS].

The endomorphism ring of a $R$-module $M$ will be denoted by $E(M)$, or $E_{R}(M)$ if there is danger of ambiguity.

A valuation domain $R$ is said to be totally branched discrete if the following two conditions are satisfied:

i) $\operatorname{Spec}(R)$ is well-ordered by the reverse inclusion, that is $\operatorname{Spec}(R)=\left\{P_{\alpha}: \alpha \leq \lambda\right\}$ where $\lambda$ is an ordinal and $P_{\alpha} \supset P_{\beta}$ for $\alpha<\beta \leq \lambda$; note that $P_{0}=P, P_{\lambda}=\{0\}$.

ii) if $J \supset H$ are two consecutive prime ideals of $R$, then $R_{J} / H$ is a DVR.

We refer to [Z] for various characterisations of totally branched discrete valuation domains. We just recall the following ones, which we shall need in the sequel: $R$ is totally branched discrete if and only if, for every $J \in \operatorname{Spec}(R), J R_{J}$ is a principal ideal of the valuation domain $R_{J}$ or, equivalently if every ideal of $R$ is isomorphic to a prime ideal.

It is worth noting a consequence of the second characterisation above: a totally branched discrete valuation domain $R$ is maximal if and only if $R / J$ is complete (in its valuation topology) for every $J \in \operatorname{Spec}(R)$ (this last property is often referred to by saying that $R$ is complete by stages). In fact, a valuation domain $R$ is maximal if and only if $R / I$ is complete for every ideal $I$ of $R$; on the other hand, by Proposition 2.2. of [SZ], if $I_{1}$ and $I_{2}$ are isomorphic ideals of $R$, then $R / I_{1}$ is complete if and only if $R / I_{2}$ is also, and thus the above assertion follows.

We shall also use the following property of a totally branched discrete valuation domain: for all nonzero prime ideal $P_{\alpha}$ of $R$, we have $R_{P_{\alpha}} \cong P_{\alpha}$.

The next two lemmas hold for any (commutative) ring $R$. Thus we state them in the general form.

Lemma 1.1. Let $R$ be any ring, and let $M, N$ be $R$-modules with isomorphic endomorphism rings; let $\Phi: E(M) \rightarrow E(N)$ be such an isomorphism. If $M=$ $M_{0} \oplus M_{1}$ and $\pi: M \rightarrow M_{0}$ is the canonical projection, then $N_{0}=\Phi(\pi) N$ is a direct summand of $N$ and we have $E\left(M_{0}\right) \cong E\left(N_{0}\right)$.

The proof of the above lemma is straightforward. 
Lemma 1.2. Let $R$ be any ring, and let $M, N$ be $R$-modules with isomorphic endomorphism rings; let $\Phi: E(M) \rightarrow E(N)$ be such an isomorphism. Suppose that $M=M_{0} \oplus M^{\prime}$, where $M_{0}$ is free of rank one; let $\pi: M \rightarrow M_{0}$ be the canonical projection and suppose that $\Phi(\pi) N=N_{0}$ is free. Then $M \cong N$.

Proof. First note that the hypotheses and Lemma 1.1 imply that $E\left(N_{0}\right) \cong E\left(M_{0}\right)=$ $R$, so that $N_{0} \cong R$, since $N_{0}$ is free. We have $M_{0}=R x_{0}, N_{0}=R y_{0}$, for suitable $x_{0} \in M, y_{0} \in N$. For each $x \in M$, let us consider the endomorphism $f_{x}$ of $M$ defined by

$$
f_{x}: r x_{0}+m^{\prime} \mapsto r x, \quad r \in R, m^{\prime} \in M^{\prime} ;
$$

note that $f_{x} \pi=f_{x}$ for all $x \in M$. The map $M \rightarrow E(M), x \mapsto f_{x}$ is an $R$ homomorphism, since it is easily checked that $f_{x+x^{\prime}}=f_{x}+f_{x^{\prime}}$ and $f_{r x}=r f_{x}$, for all $x, x^{\prime} \in M, r \in R$. Let us define a map $\psi: M \rightarrow N$ by

$$
\psi(x)=\Phi\left(f_{x}\right)\left(y_{0}\right), \quad x \in M
$$

Since $x \mapsto f_{x}$ is a homomorphism, it follows that $\psi$ is a homomorphism, too. To complete the proof, we have to show that $\psi$ is injective and surjective. Suppose that $\psi(x)=0$. By Lemma 1.1, $N_{0}$ is a summand of $N$, say $N=N_{0} \oplus N^{\prime}$; let $s y_{0}+n^{\prime}\left(s \in R, n^{\prime} \in N^{\prime}\right)$ be an arbitrary element of $N$. From $f_{x}=f_{x} \pi$ we get

$$
\Phi\left(f_{x}\right)\left(s y_{0}+n^{\prime}\right)=\Phi\left(f_{x}\right) \Phi(\pi)\left(s y_{0}+n^{\prime}\right)=s \Phi\left(f_{x}\right)\left(y_{0}\right)=s \psi(x)=0 .
$$

We deduce that $\Phi\left(f_{x}\right)=0$, whence $f_{x}=0$, since $\Phi$ is injective. Finally $f_{x}=0$ implies $f_{x}\left(x_{0}\right)=x=0$, and we conclude that $\psi$ is injective. Let us now pick any $y \in N$; let $g \in E(N)$ be defined by $g: s y_{0}+n^{\prime} \mapsto s y\left(s \in R, n^{\prime} \in N^{\prime}\right)$. Note that $g \Phi(\pi)=g$. Let $f=\Phi^{-1}(g) \in E(M)$; then $f \pi=f$ implies that $f=f_{x}$, where $x=f\left(x_{0}\right)$. We conclude that

$$
\psi(x)=\Phi\left(f_{x}\right)\left(y_{0}\right)=g\left(y_{0}\right)=y
$$

so that $\psi$ is surjective, as desired.

Lemma 1.3. Let $M$ be a separable torsion-free module over the totally branched discrete valuation domain $R$. Then there exists a prime ideal $J$ of $R$ such that $M$ has a rank-one direct summand isomorphic to $R_{J}$, and $J$ is maximal with respect to this property; moreover $R_{J}$ is the largest localization of $R$ such that $M$ is a $R_{J}$-module.

Proof. Let $\operatorname{Spec}(R)=\left\{P_{\alpha}: \alpha \leq \lambda\right\}$. Let us first suppose that $M$ is divisible. In this case every rank-one summand of $M$ is isomorphic to $Q=R_{\{0\}}$, and $M$ is naturally a $Q$-vector space. Assume now that $M$ is not divisible. Then it must contain a reduced direct summand of rank one. Every rank-one reduced $R$-module is isomorphic to an ideal of $R$, whence it is isomorphic to some $P_{\alpha} \neq 0$, since $R$ is totally branched discrete; recall also that $R_{P_{\alpha}} \cong P_{\alpha}$. Choose $\beta$ minimal such that $M$ has a summand isomorphic to $P_{\beta}$, and set $J=P_{\beta}$. Then, whenever $P_{\alpha}$ is isomorphic to a summand of $M$, we have $\beta \leq \alpha$, whence $J \supseteq P_{\alpha}$, so that $J$ is maximal with respect to the property that $R_{J} \cong J$ is isomorphic to a direct summand of $M$. If now $R_{P_{\alpha}} \supsetneqq R_{J}$, then $R_{J}$ is not a $R_{P_{\alpha}}$-module, and so neither is $M$. It remains to show that $M$ is an $R_{J}$-module. We have to verify that, for any 
given $x \in M$ and $q \in R_{J}$, we have $q x \in M$. In fact, since $M$ is separable, $x$ belongs to a rank-one summand $L$ of $M$, isomorphic to $P_{\alpha}$, say. Since $J \supseteq P_{\alpha}$, then $P_{\alpha} \cong L$ is an $R_{J}$-module. We conclude that $q x \in L \subseteq M$, as desired.

Modifying the terminology introduced by May [M], we say that a class of modules is endomorphically unique if, for any modules $M$ and $N$ in the class, $E(M) \cong E(N)$ implies $M \cong N$.

We are now in the position to prove the main result of the first section.

Theorem 1.4. Let $R$ be a valuation domain. The separable torsion-free $R$-modules are endomorphically unique if and only if $R$ is totally branched discrete.

Proof. $(\Rightarrow)$ Assume that $R$ is not totally branched discrete. Then there exists $J \in \operatorname{Spec}(R)$ such that $J R_{J}$ is not a principal ideal of $R_{J}$. It is known that $E_{R_{J}}\left(J R_{J}\right) \cong E_{R_{J}}\left(R_{J}\right) \cong R_{J}$, so that we also have $E_{R}\left(R_{J}\right) \cong E_{R}\left(J R_{J}\right)$. On the other hand, $R_{J}$ and $J R_{J}$ are not isomorphic $R$-modules, otherwise there would exist $s \in R$ such that $J R_{J}=s R_{J}$, which implies that $J R_{J}$ is a principal ideal of $R_{J}$, contrary to our assumption. We conclude that the class of separable torsion-free $R$-modules is not endomorphically unique.

$(\Leftarrow)$ Let $M$ and $N$ be separable torsion-free modules over the totally branched discrete valuation domain $R$, and assume that there exists a ring isomorphism $\Phi: E(M) \rightarrow E(N)$. Our end is to show that $M \cong N$. Let $\operatorname{Spec}(R)=\left\{P_{\alpha}: \alpha \leq \lambda\right\}$. By Lemma 1.3, there exist $\beta \leq \lambda$, minimal such that $M$ has a summand isomorphic to $R_{P_{\beta}}$, and $\beta^{\prime} \leq \lambda$, minimal such that $N$ has a summand isomorphic to $R_{P_{\beta^{\prime}}}$. Then $\beta=\beta^{\prime}$. In fact, we may assume, without loss, that $\beta \leq \beta^{\prime}$. Then we may write $M=X \oplus Y$, where $X \cong P_{\beta}$. Let $\pi: M \rightarrow X$ be the canonical projection. In view of Lemma $1.1, X^{\prime}=\Phi(\pi) N$ is a summand of $N$ such that $E\left(X^{\prime}\right) \cong E(X) \cong E\left(R_{P_{\beta}}\right)$. Then $X^{\prime}$ is indecomposable, whence $X^{\prime}$ has rank one, since it is separable. It follows that $X^{\prime} \cong R_{P_{\beta}}$, and so $\beta=\beta^{\prime}$, by the minimality of $\beta^{\prime}$. Then, from Lemma 1.3, we see that $M$ and $N$ are both $R_{P_{\beta}}$-modules. Further, in view of torsion-freeness, the $R$-endomorphisms of $M$ coincide with its $R_{P_{\beta}}$-endomorphisms, and the same holds for $N$. Since we have $X \cong R_{P_{\beta}} \cong \Phi(\pi) N=X^{\prime}$, we are in the position to apply Lemma 1.2 to the $R_{P_{\beta}}$-modules $M$ and $N$; we conclude that $M$ and $N$ are isomorphic as $R_{P_{\beta}}$-modules, and so also as $R$-modules.

\section{§2. AUTOMORPHISM GROUPS}

Let us introduce a property of a valuation domain $R$. We say that the automorphism groups determine the rank-one $R$-modules if, for all $J_{1}, J_{2}, R$-modules of rank one, $\operatorname{Aut}\left(J_{1}\right) \cong \operatorname{Aut}\left(J_{2}\right)$ implies $J_{1} \cong J_{2}$.

Of course, the above property makes sense only if $R$ is totally branched discrete, otherwise, as we have seen in the proof of Theorem 1.4, there exists a prime ideal $J$ of $R$ with $J \nRightarrow R_{J}$, but $E(J) \cong E\left(R_{J}\right)$, whence, a fortiori, $\operatorname{Aut}(J) \cong \operatorname{Aut}\left(R_{J}\right)$. Let us also note that when $R$ is a DVR, then the automorphism groups determine the rank-one $R$-modules, since these latter are isomorphic either to $\{0\}, R$, or $Q$.

The following example shows that there exist totally branched discrete valuation domains which are not DVR yet enjoy the above property, and others which do not satisfy it.

EXAMPLE 2.1. Let $D$ be a complete DVR of characteristic 0 , with maximal ideal tD and field of fractions $K$, and let $X$ be an indeterminate over $K$. Let us consider 
the following valuation domain

$$
R=D+X K[[X]],
$$

consisting of formal power series over $K$, with constant term lying in $D . R$ is a totally branched discrete valuation domain of Krull dimension 2; the prime ideals are $P \supset J \supset\{0\}$, where $P=t R$ and $J=X K[[X]]$ (the verifications of these facts are standard). Then any rank-one $R$-module is either isomorphic to one of $R, R_{J}=K[[X]]$ or $Q$. We have that $\operatorname{Aut}(R) \cong U(R)$, $\operatorname{Aut}\left(R_{J}\right) \cong U\left(R_{J}\right)$ and $\operatorname{Aut}(Q) \cong U(Q)$. Observe that every unit of $R_{J}$ may be written in the form

$$
a(1+X f(X)), \quad \text { where } a \in K \backslash\{0\}, f(x) \in K[[X]],
$$

while every unit of $R$ may be written in the form

$$
b(1+X g(X)), \quad \text { where } b \in U(D), g(x) \in K[[X]] .
$$

Finally, any nonzero element of $U(Q)$ is of the form $X^{m} \theta$ for suitable $\theta \in U\left(R_{J}\right)$ and $m \in \mathbb{Z}$. Let $G=\{1+X f(X): f(X) \in K[[X]]\}$. Since $K$ has zero characteristic it follows from Hensel's Lemma that for every $\eta \in G, n \in \mathbb{N}$, there exists $\delta \in G$ such that $\delta^{n}=\eta$. We deduce that the multiplicative group $G$ is divisible and torsion-free, whence $G$ is isomorphic to the additive group $\mathbb{Q}^{(\rho)}$ where $\rho=|K|^{\aleph_{0}}$. We conclude that $\operatorname{Aut}(R) \cong \operatorname{Aut}\left(R_{J}\right)$ if and only if $U(K) \times \mathbb{Q}^{(\rho)} \cong U(D) \times \mathbb{Q}^{(\rho)}$. Moreover we have $\operatorname{Aut}(Q) \cong \mathbb{Z} \times \operatorname{Aut}\left(R_{J}\right)$.

Let us now set $D=\hat{\mathbb{Z}}_{p}$ (the $p$-adic integers, with $p \neq 2$ ), so that $K=\hat{\mathbb{Q}}_{p}$. It is known (see $[\mathrm{F}]$, Theorem 127.5) that

$$
U\left(\hat{\mathbb{Q}}_{p}\right) \cong \mathbb{Z} \times U\left(\hat{\mathbb{Z}}_{p}\right) \cong \mathbb{Z} \times \mathbb{Z}(p-1) \times \hat{\mathbb{Z}}_{p} .
$$

Then we have $U\left(\hat{\mathbb{Q}}_{p}\right) \times \mathbb{Q}^{(\rho)} ¥ U\left(\hat{\mathbb{Z}}_{p}\right) \times \mathbb{Q}^{(\rho)}$, since $\mathbb{Z}(p-1) \times \hat{\mathbb{Z}}_{p} \times \mathbb{Q}^{(\rho)}$ has no free summands. Moreover $U(Q) \cong \mathbb{Z} \times U\left(R_{J}\right) \not U\left(R_{J}\right)$ (to see this it is enough to tensor by $\mathbb{Z}_{q}$ with $\left.q \neq p\right)$ and $U(Q) \neq U(R)$. This shows that if $R=\hat{\mathbb{Z}}_{p}+X \hat{\mathbb{Q}}_{p}[[X]]$, then the automorphism groups determine the rank-one $R$-modules.

On the other hand, if we set $D=\mathbb{Q}[[Y]]$ ( $Y$ another indeterminate, algebraically independent of $X$ ), we get $U(K)=\left\{Y^{m} g: m \in \mathbb{Z}, g \in U(D)\right\}$, whence $U(K) \cong$ $\mathbb{Z} \times U(D)$. Since any element of $U(D)$ is of the form $q(1+Y h(Y)), q \in \mathbb{Q} \backslash\{0\}$, $h(Y) \in D$, we have $U(D) \cong U(\mathbb{Q}) \times H$, where $H=\{1+Y h(Y): h(Y) \in D\}$. Since $U(\mathbb{Q})$ is a free $\mathbb{Z}$-module of countable rank, we see that

$$
U(D) \cong U(\mathbb{Q}) \times H \cong \mathbb{Z} \times U(\mathbb{Q}) \times H \cong U(K) .
$$

We conclude that, when $R=\mathbb{Q}[[Y]]+X \mathbb{Q}((Y))[[X]]$, the rank-one $R$-modules are not determined by their automorphism groups. Note that in this situation we also have $U(Q) \cong \mathbb{Z} \times U\left(R_{J}\right) \cong U\left(R_{J}\right) \cong U(R)$.

Let us remark that all the valuation domains constructed in Example 2.1 turn out to be maximal, since they are "complete by stages" (cf. the first section). Similar examples may be constructed without using maximality.

From now on we shall adopt a notation reminiscent of that in $[\mathrm{CG}]$, to facilitate comparison with that paper.

Let $g$ be an automorphism of $R$; a $g$-semi-isomorphism from the $R$-module $G$ onto the $R$-module $\widetilde{G}$ is an additive bijection from $G$ onto $\widetilde{G}$, semi-linear with respect to $g$. In such case, $G$ is said to be $g$-semi-isomorphic to $\widetilde{G}$.

In what follows we assume that 2 is a unit of $R$; equivalently, the residue field $R / P$ has characteristic $\neq 2$. 
Theorem 2.2. Let $R$ be a totally branched discrete valuation domain, with $\operatorname{Spec}(R)=$ $\left\{P_{\alpha}: \alpha \leq \lambda\right\}$. Let us suppose that 2 is a unit of $R$ and that the automorphism groups determine the rank-one $R$-modules. Let $G, \widetilde{G}$ be separable torsion-free $R$-modules such that there is an isomorphism $\Phi: A u t(G) \rightarrow A u t(\widetilde{G})$. Then the following hold: a) if $\beta, \beta^{\prime} \leq \lambda$ are, respectively, minimal such that $G$ has a summand isomorphic to $R_{P_{\beta}}$ and $\widetilde{G}$ has a summand isomorphic to $R_{P_{\beta^{\prime}}}$, then $\beta=\beta^{\prime}$;

b) $G$ and $\widetilde{G}$ are $R_{P_{\beta}}$-modules;

c) $\Phi$ determines an automorphism $g$ of $R_{P_{\beta}}$ such that either $G$ is g-semi-isomorphic to $\widetilde{G}$ as $R_{P_{\beta}}$-modules, or $G$ is g-semi-isomorphic to $\operatorname{Hom}\left(\widetilde{G}, R_{P_{\beta}}\right)$ and $\widetilde{G}$ is $g^{-1}$ semi-isomorphic to $\operatorname{Hom}\left(G, R_{P_{\beta}}\right)$, as $R_{P_{\beta}}$-modules.

Proof. Let us write $G=L \oplus H$, where $L$ is rank-one and $L \cong R_{P_{\beta}}$ with $\beta$ minimal. Since 2 is a unit of $R$, the mapping $\epsilon \mapsto(1+\epsilon) / 2$ is a bijection from the set of all involutions in $\operatorname{Aut}(G)$ to the set of all idempotents in $E(G)$. This means that $L=\operatorname{Ker}(1-\epsilon)$ for a suitable involution $\epsilon \in \operatorname{Aut}(G)$ (cf. [CG]); $\epsilon$ is said to be extremal, when, as in the present case, the corresponding summand $L$ is indecomposable.

Corresponding to the decomposition $G=L \oplus H$, every automorphism of $G$ has a matrix representation

$$
\left(\begin{array}{ll}
\xi_{11} & \xi_{12} \\
\xi_{21} & \xi_{22}
\end{array}\right)
$$

with $\xi_{i j} \in \operatorname{Hom}\left(G_{i}, G_{j}\right)$, where we have written $G_{1}=L, G_{2}=H$. In particular, absorbing a sign into $\epsilon$ if necessary, we have

$$
\epsilon=\left(\begin{array}{cc}
-1 & 0 \\
0 & 1
\end{array}\right)
$$

With respect to the fixed decomposition let $\Delta$ and $\nabla$ denote respectively all lower and all upper unitriangular matrices

$$
\left(\begin{array}{ll}
1 & 0 \\
\sigma & \mathbf{1}
\end{array}\right) \text { and }\left(\begin{array}{ll}
1 & \tau \\
0 & 1
\end{array}\right)
$$

As remarked in [CG], the image of the extremal involution $\epsilon$ is an extremal involution $\hat{\epsilon} \in \operatorname{Aut}(\widetilde{G})$, so we have a corresponding direct decomposition

$$
\widetilde{G}=\widetilde{L} \oplus \widetilde{H}
$$

where $\widetilde{L}$ has $\operatorname{rank} 1 ; \widetilde{\Delta}$ and $\widetilde{\nabla}$ now have the obvious meaning in $\operatorname{Aut}(\widetilde{G})$.

We now proceed by steps.

STEP 1: $Z($ Aut $G)$ (and similarily $Z($ Aut $\widetilde{G})$ ) consists of the matrices of the form

$$
\left(\begin{array}{cc}
a & 0 \\
0 & a \mathbf{1}
\end{array}\right) \quad \text { where } a \in \operatorname{Aut}(L)=U\left(R_{P_{\beta}}\right) .
$$

In fact, let $\left(\begin{array}{ll}a & b \\ c & d\end{array}\right) \in Z($ Aut $G)$. Since 2 is a unit of $R$, the matrix $\left(\begin{array}{ll}2 & \xi \\ 0 & \mathbf{1}\end{array}\right)$ lies in $\operatorname{Aut}(G)$, for every $\xi \in \operatorname{Hom}(L, H)$. The equality

$$
\left(\begin{array}{ll}
a & b \\
c & d
\end{array}\right)\left(\begin{array}{ll}
2 & \xi \\
0 & \mathbf{1}
\end{array}\right)=\left(\begin{array}{ll}
2 & \xi \\
0 & \mathbf{1}
\end{array}\right)\left(\begin{array}{ll}
a & b \\
c & d
\end{array}\right)
$$


gives rise to the following equalities

$$
2 c=c, \quad a \xi+b=2 b+\xi d
$$

these at once yield $c=0$ and $b=0$ (for $\xi=0$ ). Then $a \xi=\xi d$ for all $\xi \in \operatorname{Hom}(L, H)$; our end is to prove that $d=a \mathbf{1}$. Since automorphisms are represented by matrices, we make the maps operate on the right of the elements of $G, \widetilde{G}$. By contradiction, let us suppose that there exists $x \in H$ such that $x d=x^{\prime} \neq a x$. Recall that $L=R_{P_{\beta}} x_{0}$ for a suitable $x_{0} \in G$. By Lemma $1.3 G$ is an $R_{P_{\beta}}$-module, so that there exists an $R_{P_{\beta}}$-homomorphism $\xi: L \rightarrow H$ such that $\xi: x_{0} \mapsto x$. But then $x_{0} a \xi=a x \neq x^{\prime}=x d=x_{0} \xi d$; this gives our required contradiction.

STEP 2: $L \cong \widetilde{L}$.

In view of the hypothesis on $R$, it is enough to show that $\operatorname{Aut}(L) \cong \operatorname{Aut}(\widetilde{L})$. STEP 1 implies that the isomorphism $\Phi$ induces a map $Z(\operatorname{Aut} G) \rightarrow Z($ Aut $\widetilde{G})$ given by

$$
\Phi:\left(\begin{array}{cc}
a & 0 \\
0 & a \mathbf{1}
\end{array}\right) \mapsto\left(\begin{array}{cc}
\rho(a) & 0 \\
0 & \rho(a) \mathbf{1}
\end{array}\right)
$$

It is then clear that the map $a \mapsto \rho(a)$ is an isomorphism of $\operatorname{Aut}(L)$ onto $\operatorname{Aut}(\widetilde{L})$, as desired.

From STEP 2 and by the symmetry between $G$ and $\widetilde{G}$, we get that $\beta$ is also the minimal ordinal such that $\widetilde{G}$ has a direct summand isomorphic to $R_{P_{\beta}}$, that is, in our notation, $\beta=\beta^{\prime}$. It follows that $G, \widetilde{G}$ are both $R_{P_{\beta}}$-modules, and this settles points a) and b) of our statement.

Since $G, \widetilde{G}$ are torsion-free, we also have that the endomorphisms of $G$ as an $R$-module and those as an $R_{P_{\beta}}$-module coincide, and the same for $\widetilde{G}$. It follows that $\operatorname{Aut}(G) \cong \operatorname{Aut}(\widetilde{G})$ when $G, \widetilde{G}$ are viewed as $R_{P_{\beta}}$-modules. It is then clear that we may thus assume, without loss, that $L \cong \widetilde{L} \cong R$. This assumption will be standing for the rest of the proof. In particular, we emphasize that if $G$ is divisible, then $\beta=\lambda, R_{P_{\lambda}}=R_{\{0\}}=Q$ and $G, \widetilde{G}$ will be regarded as $Q$-vector spaces.

The following step, proved in [CG], is crucial.

$\operatorname{STEP} 3$. Either $(\mathrm{i}) \Phi(\Delta)=\widetilde{\Delta}$ and $\Phi(\nabla)=\widetilde{\nabla}$, or (ii) $\Phi(\Delta)=\widetilde{\nabla}$ and $\Phi(\nabla)=\widetilde{\Delta}$.

The above result is proved in $[\mathrm{CG}]$ (Lemma 3 ) in the case of $\hat{\mathbb{Z}}_{p}$-modules, but their proof is purely group-theoretical, and so it goes through also in our case. All they need of module theory is that the centre of the endomorphism algebra of a torsion-free separable $R$-module containing a free summand, consists of the scalar multiplications: this fact can be verified arguing as in STEP 1.

STEP 4. The image under $\Phi$ of an automorphism of the form $\left(\begin{array}{ll}u & 0 \\ 0 & \mathbf{1}\end{array}\right), u \in U(R)$, has the form $\left(\begin{array}{cc}r(u) & 0 \\ 0 & s(u) \mathbf{1}\end{array}\right), r(u), s(u) \in U(R)$. Moreover, the map $f: u \mapsto$ $r(u) / s(u)$ is a multiplicative homomorphism of $U(R)$.

Since $\left(\begin{array}{ll}u & 0 \\ 0 & \mathbf{1}\end{array}\right)$ belongs to $Z C(\epsilon)$, then $\Phi\left(\begin{array}{ll}u & 0 \\ 0 & \mathbf{1}\end{array}\right)$ belongs to $Z C(\Phi(\epsilon))$, and therefore it has the required form, as proved in [CG]. Since $\Phi$ is multiplicative, for $u, v \in U(R)$ we have $r(u v)=r(u) r(v), s(u v)=s(u) s(v)$, whence $f(u v)=f(u) f(v)$. 
Let us now consider any element $\left(\begin{array}{ll}1 & \sigma \\ 0 & \mathbf{1}\end{array}\right) \in \Delta$, where $\sigma \in \operatorname{Hom}(L, H)$. Now, if we are in the situation of case (i) of STEP 3, its image under $\Phi$ will have the form $\left(\begin{array}{cc}1 & \phi(\sigma) \\ 0 & \mathbf{1}\end{array}\right)$, with $\phi(\sigma) \in \operatorname{Hom}(\widetilde{L}, \widetilde{H})$. Otherwise, if we are in case (ii) of STEP 3, the image will be of the form $\left(\begin{array}{cc}1 & 0 \\ \psi(\sigma) & \mathbf{1}\end{array}\right)$, where $\psi(\sigma) \in \operatorname{Hom}(\widetilde{H}, \widetilde{L})$. It is immediate to see that

$$
\phi: \operatorname{Hom}(L, H) \rightarrow \operatorname{Hom}(\widetilde{L}, \widetilde{H}) ; \quad \psi: \operatorname{Hom}(L, H) \rightarrow \operatorname{Hom}(\widetilde{H}, \widetilde{L})
$$

are additive maps. In view of STEP $3, \phi, \psi$ turn out to be bijective.

STEP 5. Let $f$ be the map defined in STEP 4. The map $g: R \rightarrow R$ defined by $g(u)=f(u)$, for $u \in U(R)$ and $g(r)=f(r-1)+1$ for $r \in P$ is an automorphism of $R$.

Let us assume to be in case (i) of STEP 3; the argument for case (ii) is similar. We have the additive map $\phi$ defined above; let us first see that $\phi(r \sigma)=g(r) \phi(\sigma)$, for all $r \in R$ and $\sigma \in \operatorname{Hom}(L, H)$. In fact, let us first assume that $r \in P$; applying $\Phi$ and recalling STEP 4, we get

(1)

$$
\begin{aligned}
\left(\begin{array}{cc}
1 & r \sigma \\
0 & \mathbf{1}
\end{array}\right) & =\left(\begin{array}{cc}
r-1 & 0 \\
0 & \mathbf{1}
\end{array}\right)\left(\begin{array}{ll}
1 & \sigma \\
0 & \mathbf{1}
\end{array}\right)\left(\begin{array}{cc}
1 /(r-1) & 0 \\
0 & \mathbf{1}
\end{array}\right)\left(\begin{array}{cc}
1 & \sigma \\
0 & \mathbf{1}
\end{array}\right) \\
& \mapsto\left(\begin{array}{cc}
1 & f(r-1) \phi(\sigma) \\
0 & \mathbf{1}
\end{array}\right)\left(\begin{array}{cc}
1 & \phi(\sigma) \\
0 & \mathbf{1}
\end{array}\right)=\left(\begin{array}{cc}
1 & (f(r-1)+1) \phi(\sigma) \\
0 & \mathbf{1}
\end{array}\right) .
\end{aligned}
$$

In a similar, slightly simpler, way, we see that $\phi(u \sigma)=g(u) \phi(\sigma)$ for all $u \in U(R)$, and we are done. Let us now show that $g$ is additive. Let $0 \neq \sigma \in \operatorname{Hom}(L, H)$; for all $r, s \in R$ we have $r \sigma+s \sigma \mapsto g(r) \phi(\sigma)+g(s) \phi(\sigma)$, since $\phi$ is additive. From $(r+s) \sigma \mapsto g(r+s) \phi(\sigma)$, we get $g(r+s)=g(r)+g(s)$, since $\sigma \neq 0, \phi$ is injective, and $G, \widetilde{G}$ are torsion-free. To see that $g$ is multiplicative, it is enough to note that $r s \sigma \mapsto g(r) \phi(s \sigma)=g(r) g(s) \phi(\sigma)$, for all $r, s, \sigma$. It remains to show that $g$ is bijective. For a fixed $\sigma \neq 0$, from $g(r)=g(s)$ it follows $\phi(r \sigma)=\phi(s \sigma)$; since $\phi$ is injective, we get $r \sigma=s \sigma$, whence $r=s$. We conclude that $g$ is injective. Now pick $t \in R$; since $\phi$ is surjective, for any fixed $\sigma$ there exists $\tau$ such that $\phi(\tau)=t \phi(\sigma)$. Applying $\Phi^{-1}$ to $t \phi(\sigma)$ we see, as in (1), that $\tau=r \sigma$, for a suitable $r \in R$; it follows that $t \phi(\sigma)=g(r) \phi(\sigma)$, whence $t=g(r)$. We conclude that $g$ is also surjective.

For an $R$-module $M$, we use the standard notation $M^{*}=\operatorname{Hom}_{R}(M, R)$. The next STEP 6 will end our proof.

STEP 6. If we are in case (i) of STEP 3, then $G$ is $g$-semi-isomorphic to $\widetilde{G}$; if we are in case (ii), then $G$ is $g$-semi-isomorphic to $\widetilde{G}^{*}$ and $\widetilde{G}$ is $g^{-1}$-semi-isomorphic to $G^{*}$.

We have seen in the proof of STEP 5 that in case (i), $\phi$ is a $g$-semi-isomorphism from $\operatorname{Hom}(L, H) \cong H$ onto $\operatorname{Hom}(\widetilde{L}, \widetilde{H}) \cong \widetilde{H}$. In a similar way, one can see that, in case (ii), $\psi$ is a $g$-semi-isomorphism from $\operatorname{Hom}(L, H)$ onto $\operatorname{Hom}(\widetilde{H}, \widetilde{L}) \cong \widetilde{H}^{*}$. Since $L \cong \widetilde{L} \cong R, L$ is automatically $g$-semi-isomorphic to $\widetilde{L}$. The desired conclusions follow easily.

We have a corollary of the preceding theorem, which essentially extends the main result in $[\mathrm{CG}]$. 
Theorem 2.3. Let $D$ be a DVR contained in $\hat{\mathbb{Z}}_{p}$. Let $G, \widetilde{G}$ be separable torsionfree $D$-modules such that $A u t(G) \cong A u t(\widetilde{G})$. Then either $G \cong \widetilde{G}$ or $G \cong \widetilde{G}^{*}$ and $\widetilde{G} \cong G^{*}$.

Proof. From $D \subseteq \hat{\mathbb{Z}}_{p}$ it follows that $p D$ is the maximal ideal of $D$, the $p$-adic topology on $D$ coincides with the topology induced by the $p$-adic topology on $\hat{\mathbb{Z}}_{p}$, and $D$ is dense in $\hat{\mathbb{Z}}_{p}$, since it necessarily contains $\mathbb{Z}_{p}$. Let us also note that, since $D$ is a DVR, it satisfies the hypothesis of Theorem 2.2 . If $G$ is divisible, then $\widetilde{G}$ is divisible too, by Theorem 2.2, so they are both vector spaces and our claim follows from the classical result by Rickart [R]. Assume now that $G$ is not divisible. Then $G$ contains a free summand. Since the identity is the unique ring automorphism of $\hat{\mathbb{Z}}_{p}$, then also $D$ has only the trivial automorphism. An application of Theorem 2.2 gives the desired conclusion.

In the notation of Theorem 2.2, one may ask if the case when $G$ is not $g$-semiisomorphic to $\widetilde{G}$ (as $R_{P_{\beta}}$-modules) can actually happen. The answer is affirmative, as shown by the next example.

EXAMPLE 2.4. Consider the two separable $\mathbb{Z}_{p}$-modules $\Pi=\prod_{\aleph_{0}} \mathbb{Z}_{p}$ and $\Sigma=$ $\bigoplus_{\aleph_{0}} \mathbb{Z}_{p}$. Of course $\Pi \neq \Sigma$, so that $E(\Pi) \neq E(\Sigma)$. However, Charles has proved in $[\mathrm{C}]$ that the rings $E(\Pi)$ and $E(\Sigma)$ are anti-isomorphic, so that the groups Aut( $(\Pi)$ and $\operatorname{Aut}(\Sigma)$ are anti-isomorphic. But then $\operatorname{Aut}(\Pi) \cong \operatorname{Aut}(\Sigma)$ (this follows from the fact that the map $x \mapsto x^{-1}$ is an anti-isomorphism of any group). Applying our Theorem 2.2 and recalling that $\mathbb{Z}_{p}$ has no nontrivial ring automorphisms, we see that $\Pi \cong \Sigma^{*}, \Sigma \cong \Pi^{*}$, and $\Pi \neq \Sigma$.

It is also interesting to show that the hypothesis in Theorem 2.2 that the automorphism groups determine the rank-one $R$-modules cannot be avoided.

Proposition 2.5. Let $R$ be a totally branched discrete valuation domain which contains a non-maximal prime ideal $J$ such that $U(J) \cong U(R)$. Then, for any automorphism $g$ of $R$, there are no $g$-semi-isomorphisms from $R$ onto $J$ (nor onto $J^{*}$, which is isomorphic to $J$ ).

Proof. First of all, let us note that $J \cong J^{*}$. In fact, $J^{*} \cong R_{J}$ (this is true for any prime ideal of an arbitrary valuation domain), and $R_{J} \cong J$, since $R$ is totally branched discrete. Assume for a contradiction that $\phi: R \rightarrow J$ is a $g$-semiisomorphism, for a suitable $g \in \operatorname{Aut}(R)$. Let $\phi(1)=a \in J$. Choose $d \in R \backslash J$ which is not a unit. Then $a / d \in J$, since $J$ is a prime ideal. Let $u=\phi^{-1}(a / d) \in R$. Then $\phi\left(g^{-1}(d) u\right)=g g^{-1}(d) \phi(u)=d a / d=a$ implies that $g^{-1}(d) u=1$, whence $d v=1$, where $v=g(u)$. It follows that $d$ is a unit of $R$, impossible.

Let us remark that the valuation domain $R$, constructed in Example 2.1, such that the automomorphism groups do not determine the rank-one $R$-modules, fulfills the hypotheses of the above proposition. In fact $R$ is of the form $R=D+X K[[X]]$, where $J=X K[[X]]$ is such that $U(R) \cong U(J)$. Then Proposition 2.5 shows that the conclusions of Theorem 2.2 do not hold if we set $G=R$ and $\widetilde{G}=J$.

We end our paper with a discussion of the limitations of the method employed in the proof of Theorem 2.2.

$R E M A R K$ 2.6. We shall adopt the notation of Theorem 2.2. We start exhibiting an example of an automorphism $g$ of $R_{P_{\beta}}$ such that $g(R) \not \subset R$. 
Let $T=K[[X]]$, where $K$ is the field $K=\mathbb{Q}(y, z)$ (y,z are indeterminates). Let $D=\mathbb{Q}[y, z]_{(y)}$; then $D$ is a valuation domain of $K$ (discrete of rank one). Let us now consider the valuation domain

$$
R=D+X K[[X]]
$$

Note that $T=R_{J}$, where $J=X K[[X]]$. Let $\varphi: K \rightarrow K$ be the automorphism which extends the assignments $y \mapsto z$ and $z \mapsto y$. Then the map $g: T \rightarrow T$ defined by

$$
g: \sum a_{i} X^{i} \mapsto \sum \varphi\left(a_{i}\right) X^{i}
$$

is an automorphism of $T$. However, note that $\varphi D=\mathbb{Q}[y, z]_{(z)} \not \subset D$, since, for instance, $y$ is a unit in $\varphi D$ but not in $D$. It follows that

$$
g(R)=\varphi D+X K[[X]] \not \subset R
$$

in other words, $\left.g\right|_{R}$ is not an automorphism of $R$.

Let us now apply the procedure in the proof of Theorem 2.2 to the following situation: $R$ is the above defined valuation domain; $G=T \oplus T=\widetilde{G}$ ( $T$ as above); $\Phi$ is the isomorphism of $\operatorname{Aut}(G)$ onto $\operatorname{Aut}(\widetilde{G})$ which maps the $2 \times 2$ matrix, invertible over $T,\left(\begin{array}{ll}a & b \\ c & d\end{array}\right)(a, b, c, d \in T)$ to $\left(\begin{array}{cc}g(a) & g(b) \\ g(c) & g(d)\end{array}\right)$, where $g$ is the above defined automorphism of $T$.

Here, in the notation of Theorem 2.2, $P_{\beta}=J, T=R_{P_{\beta}}$, and, following the proof, the automorphism of $R_{P_{\beta}}$, determined by $\Phi$, turns out to be the same as the $g$ defined above, whose restriction to $R$ is not an automorphism of $R$. From the theorem we just conclude that $G$ is $g$-semi-isomorphic to $\widetilde{G}$, as $R_{P_{\beta}}$-modules, but not as $R$-modules.

Thus a straight application of the method in Theorem 2.2, based on the map $\Phi$, is unable to show not only that $G \cong \widetilde{G}$, but even that there is a semi-linear isomorphism from $G$ to $\widetilde{G}$ as $R$-modules.

In the general situation, we have a stronger result if $G$ satisfies the following property:

$\left.{ }^{*}\right)$ for every $f \in \operatorname{Aut}\left(R_{P_{\beta}}\right)$ there exists a $f$-semi-isomorphism from $G$ onto $G$, as $R_{P_{\beta}}$-modules.

With such a property available, we could apply it, in Theorem 2.2, setting $f=$ $g^{-1}$, to obtain $G \cong \widetilde{G}$ or $G \cong \operatorname{Hom}\left(\widetilde{G}, R_{P_{\beta}}\right)$, as $R_{P_{\beta}}$-modules, and so also as $R$-modules. Note that $G$ satisfies property $\left(^{*}\right)$ if it is a product of copies of $R$.

We are not able to say if property $\left(^{*}\right)$ is satisfied by any separable torsion-free $R$-module, or, more generally, if Theorem 2.2 can be improved replacing semiisomorphism with isomorphism.

\section{REFERENCES}

[B] R. Baer, Linear Algebra and Projective Geometry, Academic Press, New York, 1952.

[C] B. Charles, Méthodes topologique en théorie des groupes abéliens, Proc. Colloq. Abelian Groups, Budapest (1964), 29-42.

[CG] A.L.S. Corner and B. Goldsmith, Isomorphic automorphism groups of p-adic modules, to appear.

[F] L. Fuchs, Infinite Abelian Groups, Academic Press, New York and London, 1970 and 1973. 
[FS] L. Fuchs and L. Salce, Modules over Valuation Domains, Marcel Dekker, New York and Basel, 1985.

[H] C.J. Hauptfleisch, Torsion-free abelian groups with isomorphic endomorphism rings, Arch. Math. 24 (1973), 269-273.

[K] I. Kaplansky, Infinite Abelain Groups, University of Michigan Press, Ann Arbor, 1954.

[Le] H. Leptin, Abelsche p-gruppen und ihre Automorphismengruppen, Math. Z. 73 (1960), 235253.

[Li] H. Liebert, Isomorphic automorphism groups of primary abelian groups, in Abelian Group Theory, Proceedings of the 1985 Oberwolfach Conference, Gordon and Breach, New York (1987), 9-31.

[M] W. May, Endomorphism algebras of not necessarily cotorsion-free modules, Contemporary Math. Vol 130, AMS (1992), 257-264.

[R] C.E. Rickart, Isomorphic groups of linear transformations, Amer. J. Math. 72 (1950), 451464.

[SZ] L. Salce and P. Zanardo, Some cardinal invariants for valuation domains, Rend. Sem. Mat. Padova 74 (1985), 205-217.

[W] K.J. Wolfson, Isomorphisms of the endomorphism rings of torsion-free modules, Proc. Amer. Math. Soc. 13 (1962), 712-714.

[Z] P. Zanardo, Valuation domains without pathological modules, J. Algebra 96 (1985), 1-8.

B. Goldsmith, Dublin Institute of Technology, Kevin Street, Dublin 8, Ireland E-mail address: BGOLDSMITH@ DIT.IE

Paolo Zanardo, Dipartimento di Matematica Pura e Applicata, Via Belzoni 7, 35131 Padova, Italy.

E-mail address: PZANARDO@ MATH.UNIPD.IT 